Intrinsic and Extrinsic Magnetic Properties of the Naturally Layered Manganites

A. Berger, J.F. Mitchell, D.J. Miller, J.S. Jiang, and S.D. Bader

Materials Science Division

Argonne National Laboratory, Argonne, IL 60439

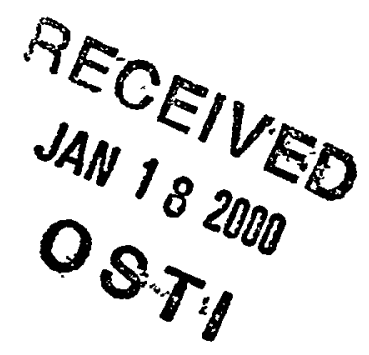

Proc. 46th Int. Symp. of the American Vacuum Society, Seattle, WA, Oct. 25-29, 1999 (to be published by Jour. Vac. Sci. and Technol.) \#W-31-109-ENG-38. 


\section{DISCLAIMER}

This report was prepared as an account of work sponsored by an agency of the United States Government. Neither the United States Government nor any agency thereof, nor any of their employees, make any warranty, express or implied, or assumes any legal liability or responsibility for the accuracy, completeness, or usefulness of any information, apparatus, product, or process disclosed, or represents that its use would not infringe privately owned rights. Reference herein to any specific commercial product, process, or service by trade name, trademark, manufacturer, or otherwise does not necessarily constitute or imply its endorsement, recommendation, or favoring by the United States Government or any agency thereof. The views and opinions of authors expressed herein do not necessarily state or reflect those of the United States Government or any agency thereof. 


\section{DISCLAIMER}

\section{Portions of this document may be illegible in electronic image products. Images are produced from the best available original document.}




\title{
Intrinsic and Extrinsic Magnetic Properties of the Naturally Layered Manganites
}

\author{
A. Berger, J. F. Mitchell, D. J. Miller, J. S. Jiang, and S. D. Bader \\ Materials Science Division, \\ Argonne National Laboratory, Argonne, IL 60439 (USA)
}

\begin{abstract}
:
Structural and magnetic properties of the two-layered Ruddlesden-Popper phase SrO $\left(\mathrm{La}_{1}\right.$. $\left.{ }_{x} \mathrm{Sr}_{\mathrm{x}} \mathrm{MnO}_{3}\right)_{2}$ with $\mathrm{x}=0.3-0.5$ are highlighted. Intrinsic properties of these naturally layered manganites include a 'colossal' magnetoresistance, a composition-dependent magnetic anisotropy, and almost no remanence. Above the Curie temperature there is a non-vanishing extrinsic magnetization attributed to intergrowths (stacking faults in the layered structure). These lattice imperfections consist of additional or missing manganite layers, as observed in transmission electron microscopy. Their role in influencing the properties of the host material is highlighted.
\end{abstract}

Keywords: intergrowths, colossal magnetoresistance, layered materials, manganites, magnetic measurements 


\section{Introduction:}

Understanding the electronic properties of transition metal oxides has long been a challenge. These materials are rich in metal-insulator and structural transitions and can also have intriguing magnetic and superconducting properties. Presently there is renewed interest in doped manganese oxides because they exhibit a 'colossal' magnetoresistance (CMR) [1] and hold the promise of $100 \%$ electron spin polarization due to a half-metallic electronic structure. In this paper we discuss the properties of a subclass of the CMR oxides known as the naturally layered manganites. These can be envisioned, as will be shown, as naturally occurring superlattices, and their quasi-two dimensionality introduces interesting new anisotropies into the system. As with any new and complex material the initial goal is to separate intrinsic and extrinsic properties. Characterizing intrinsic properties permits the underlying physics to be identified, while separating the extrinsic properties identifies materials issues that can also play an important role.

In short, the CMR in the doped manganites is related to a temperature dependent phase transition that occurs from a paramagnetic insulator to a ferromagnetic metal. Just above this transition an applied magnetic field not only restores magnetic order (as it would in all types of ferromagnetic materials) but also stabilizes the metallic state. CMR is displayed in Fig. 1 for the two-layered variant that will be the focus of the present work.

A qualitative explanation for the simultaneous occurrence of ferromagnetism and metallic conductivity was proposed by Zener long ago; it is known as the 'double exchange' (DE) mechanism [2-4]. To describe DE we adopt a localized, purely ionic picture as the starting point and consider the crystal fields. First we describe threedimensional structures, and then the layered variants. A perovskite-type structure for $\mathrm{La}_{1}$ ${ }_{x} \mathrm{Sr}_{x} \mathrm{MnO}_{3}$ is shown in Fig. 2. The trivalent La could equally well be $\mathrm{Pr}^{3+}, \mathrm{Nd}^{3+}$ etc. and the divalent $\mathrm{Sr}$ dopant could be $\mathrm{Ca}^{2+}, \mathrm{Ba}^{2+}$ etc. They occupy type A (body centered) lattice sites as a homogeneous solid solution. The $\mathrm{O}^{2-}$ ions form interlocking (corner-sharing) octahedra surrounding the $\mathrm{Mn}$ atoms, which are on cube corner sites. In order to conserve charge neutrality, the manganese ions have a mixed valence of $\mathrm{Mn}^{3+}\left(3 \mathrm{~d}^{4}\right)$ and $\mathrm{Mn}^{4+}\left(3 \mathrm{~d}^{3}\right)$. The proportion of the two valence states depends on $\mathrm{x}$. Both states are in high-spin 
configurations dictated by strong intra-atomic Hund's rules couplings. The octahedral coordination splits the $\mathrm{Mn} 3 \mathrm{~d}$-orbitals into a lower lying $\mathrm{t}_{\mathrm{g}}$ triplet and a higher lying $\mathrm{e}_{\mathrm{g}}$ doublet state with occupations of $\mathrm{t}_{2 \mathrm{~g}}{ }^{3} \mathrm{e}_{\mathrm{g}}{ }^{1}$ and $\mathrm{t}_{2 \mathrm{~g}}{ }^{3}$ for $\mathrm{Mn}^{3+}$ and $\mathrm{Mn}^{4+}$, respectively (see Fig. 2). There are no direct $\mathrm{Mn}-\mathrm{Mn}$ interactions because of the long distance of separation. Even though the basic cubes and octahedra are distorted, near-linear Mn-O-Mn bond lengths dominate the indirect coupling of $\mathrm{Mn}$ ions mediated by the intervening oxygen. This gives rise to antiferromagnetic super-exchange interactions and competing ferromagnetic double-exchange interactions. The filled $t_{2 g}$ orbitals, which are largely responsible for the super-exchange, form a local $S=3 / 2$ spin. This local spin, is coupled (again via Hund's rules) to the spin of the $e_{g}$ electron, creating an $\mathrm{S}=2$ state on the $\mathrm{Mn}^{3+}$ ion site. The degeneracy of the two $\mathrm{e}_{\mathrm{g}}$ orbitals of $\mathrm{Mn}^{3+}$ is removed by a Jahn-Teller (JT) distortion of the oxygen octahedra, while $\mathrm{Mn}^{4+}$ is not a JT ion. The $\mathrm{O}(2 \mathrm{p})$ orbital mediates a hopping of $\mathrm{e}_{\mathrm{g}}$ electrons from a $\mathrm{Mn}^{3+}$ to an adjacent $\mathrm{Mn}^{4+}$ site. Accordingly, the name double exchange describes the simultaneous electron hopping from $\mathrm{Mn}^{3+}$ to $\mathrm{O}(2 \mathrm{p})$ and from $\mathrm{O}\left(2 \mathrm{p}\right.$ ) into an empty $\mathrm{Mn}^{4+} \mathrm{e}_{\mathrm{g}}$ state (see Fig. 3). The important feature of the double exchange mechanism which stabilizes ferromagnetism is that the effective overlap or hopping matrix element between the two neighboring $\mathrm{Mn}$ ions strongly depends on the relative orientation of the local $\mathrm{Mn}$ spins, as shown by Anderson and Hasegawa [3]. The $\mathrm{e}_{\mathrm{g}}$ electron can only delocalize, $i$.e. form a metallic state, when the two $\mathrm{Mn}$ ion spin states have at least a certain degree of alignment; hence, ferromagnetic order stabilizes the metallic state. It is this delocalization of the $\mathrm{e}_{\mathrm{g}}$ electrons that provides the condensation energy for the ferromagnetic state. Hence, the magnetism of the CMR materials is fundamentally different from that, say, of the itinerant magnets $\mathrm{Fe}, \mathrm{Ni}$ and Co. For the elemental ferromagnets a spin-split electronic band structure removes a peak in the density of states at the Fermi energy in order to lower the total energy. For the CMR materials it is the energy of delocalizing a spin-polarized $\left(e_{g}\right)$ electron (and associated polaronic couplings, as outlined below) that dominate the stabilization of the ferromagnetic state. Thus, within the DE picture, ferromagnetic coupling and metallic conductivity are intimately connected, giving us a base to understand the manganites.

Polaronic effects also play a major role in the energetics. These effects can be understood as being due to the fact that the $e_{\mathrm{g}}$ electron carries with it the JT distortion, 
especially if the hopping is slow enough, i.e. above $\mathrm{T}_{\mathrm{c}}$. Thus the double exchange is importantly renormalized by this electron-lattice coupling interaction. The Jahn-Teller effect lifts the degeneracy typically by reducing the energy of the $3 z^{2}-r^{2} e_{g}$-type orbital (as seen in Fig. 2). This also implies that changes in the lattice parameters can be expected in the vicinity of $T_{C}$, where the $e_{g}$ electrons delocalize. The local character of $\mathrm{Mn}^{3+}$ and $\mathrm{Mn}^{4+}$ ions should be largely washed out below $\mathrm{T}_{\mathrm{C}}$. Figure 2 also indicates that specific d-orbitals are preferably occupied. Due to their non-spherical shape, the occupation and orientation of orbitals on neighboring sites are not independent of each other, but can exhibit a tendency for orbital ordering. In addition, the simple DE model does not contain a Coulomb repulsion term, which is needed to explain charge ordering effects in the manganites [5].

In general, the properties of the mixed valence manganites arise from these competing interactions which can have similar energy scales. $\mathrm{DE}$ is helpful to get a first glimpse of the underlying physics, but cannot explain the full range of observed phenomena. [6] Besides the fact, that manganites are fascinating materials and exhibit a variety of unusual physical properties, they are also of technological interest due to the CMR effect and spin-polarized electronic structure. Therefore, a vast amount of the recent work has been conducted towards optimizing the CMR properties with respect to magnitude, field sensitivity, and temperature range of operation [7].

So far, most CMR studies have focused on the perovskite manganites like $\mathrm{La}_{1-x}$ $\mathrm{Sr}_{\mathrm{x}} \mathrm{MnO}_{3}$. However, Moritomo et al. demonstrated that it is possible to fabricate a layered form of these materials, in particular the $n=2$ variant of the Ruddlesden-Popper series $(\mathrm{La}, \mathrm{Sr})_{\mathrm{n}+1} \mathrm{Mn}_{\mathrm{n}} \mathrm{O}_{3 \mathrm{n}+1}$ [8]. Figure 4 illustrates the $n=1,2, \infty$ members of this family. The unit cell may be written as $\mathrm{SrO}\left(\mathrm{La}_{1-x} \mathrm{Sr}_{x} \mathrm{MnO}_{3}\right)_{\mathrm{n}}$ with $n$ being the number of $\mathrm{MnO}_{6}$ octahedral layers in each unit cell. The $n=\infty$ compound then refers to the bulk perovskite structure, whereas the $n=1$ member consists of separated $\mathrm{MnO}_{6}$ layers and does not show ferromagnetic order at accessible temperatures [9]. The bilayer structure $n=2$, however, has interesting magnetic and related properties and is presently the focus of intense research. efforts $[8,10-23]$. The unit cell consists of $\mathrm{MnO}_{6}$ octahedral bilayers which are separated by an insulating SrO rock-salt layer. The structure is tetragonal, with the ab-plane parallel to the layers of $\mathrm{MnO}_{6}[10]$. For instance, the $\mathrm{x}=0.4$ variant of this 
material is reported to have $\mathrm{a} \sim 20,000 \% \mathrm{CMR}$ with $\mathrm{H}=7 \mathrm{~T}$, and $\sim 200 \% \mathrm{CMR}$ at low fields $(0.3 \mathrm{~T})$ for $\mathrm{T}=129 \mathrm{~K}$ [8]. All of these materials (including the $n=\infty$ variety) exhibit a competition between antiferromagnetism (due to superexchange) and ferromagnetism (due to double exchange). The perovskite type manganites show antiferromagnetic order for $\mathrm{x}=0$, where double exchange is absent, while for $\mathrm{x} \approx 0.2$ 0.4 , they are ferromagnetic, and exhibit a metal-insulator transition at $T_{C}$. The $T_{C}$-values for the bilayered system are significantly reduced compared to comparably doped 3D perovskites, which can be understood as a 'finite-size' effect $\left(T_{c}\right.$ increases with $\left.n\right)$. As for the 3D systems, $T_{C}$ of the layered manganites broadens and moves appreciably in an external field $\mathrm{H}$, which in turn shifts and broadens the metal-insulator transition and causes the CMR. As already shown in Fig.1, the external field is thought to help align the Mn spins and therefore facilitate the electron delocalization via the double exchange mechanism [2].

For the layered compounds, most of the research effort has been focused on the $\mathrm{x}$ $=0.3-0.5$ concentration range. Only recently it became feasible to grow $n=2$ samples in the Sr rich $(x=0.5-1)$ composition range [24]. An overview of the known magnetic ground states is given in Fig. 5. For the $x=0.3-0.4$ range, one finds ferromagnetic order within the bilayer and for $x \geq 0.32$ also 3D ferromagnetic alignment between the bilayers. In the case of $x=0.30$, the weak inter-bilayer coupling is antiferromagnetic (AF), producing an A-type AF groundstate with moments aligned along the c-axis. For materials near half-filling $(x \approx 0.5)$ an A-type $A F$ order is found with anti-parallel alignment of the magnetic moments in the individual layers of each bilayer.

A general feature of all these layered compounds is the fact that the inter-bilayer coupling is orders of magnitudes smaller than the intra-bilayer coupling. Thus, these naturally occurring superlattices can be envisioned as constituting an array of weakly coupled ultrathin magnetic films. Therefore, they allow the study of $2 \mathrm{D}$ magnetism and other ultrathin film phenomena utilizing bulk methods such as neutron and $\mathrm{x}$-ray scattering [13, 14, 19-22]. And ultimately they possess 2D-3D crossover effects, since the metal-insulator transition takes place simultaneously in-plane and between planes, as shown in the resistivity data in Fig. 1. 
Besides the CMR effect, the bilayer phase $\mathrm{SrO}\left(\mathrm{La}_{1-\mathrm{x}} \mathrm{Sr}_{x} \mathrm{MnO}_{3}\right)_{2}$ exhibits a number of interesting structural and magnetic properties. The structural properties have been extensively investigated using $x$-ray and neutron scattering and unusually large magnetoelastic effects have been observed [13] including lattice parameter anomalies around $\mathrm{T}_{\mathrm{C}}$. Interestingly, these changes strongly depend on the doping level $\mathrm{x}$ and can even exhibit the opposite sign of the conventional Jahn-Teller deformation [13]. These lattice effects also correlate with the magnetic order, because the $\mathrm{x}=0.36$ compound, which does not show unusual thermal anomalies in the lattice constants, exhibits the highest $T_{C}$ of the layered manganites [21]. The critical behavior near $T_{c}$ has also been studied but a clear understanding is allusive probably because of anticipated crossovers close to $\mathrm{T}_{\mathrm{C}}$ [20]. For the 0.4 compound, charge density fluctuations and AF correlations also are observed in the paramagnetic state [22]. Furthermore, for the entire $x=0.3-0.4$ range an anomalous field dependence of the magnetic susceptibility has been observed, which indicates competing interactions in the paramagnetic state [25]. However, quantitative understandings are still missing. More work is necessary to explore the rich variety of phenomena found in the layered materials. In particular, the important role of polarons and dynamic $v s$ static JT effects in governing the energetics of the phase transition needs further elucidation.

Besides the possibility of unusual intrinsic effects, one has to realize that there are also significant materials issues. In particular, it has been reported that these layered materials contain intergrowths defects $[18,23]$. Certain magnetic phenomena observed in those materials are caused or influenced by these extrinsic sample imperfections which can be envisioned as an occasional stacking fault with missing or added SrO spacer layers. Thus, an awareness of the presence of intergrowths and knowledge of their extent and influence is essential in understanding the layered CMR materials. This article focuses primarily on intergrowths. After a brief discussion of experimental procedures in Sec. 2 we explore the structural properties of the intergrowths (Sec. 3). Magnetic properties are then analyzed in Sec. 4. Finally, we summarize our results in Sec. 5 and discuss the interest in intergrowth structures as atomically flat inclusions with $2 \mathrm{D}$ physical properties. 


\section{EXPERIMENTAL}

Bulk crystals of $\mathrm{La}_{2-2 x} \mathrm{Sr}_{1+2 x} \mathrm{Mn}_{2} \mathrm{O}_{7}(\mathrm{x}=0.3$ - 0.5$)$ were grown from polycrystalline rods of the same nominal composition using the traveling-floating-zone technique in an optical image furnace (NEC model SC-M15HD). The precursor rods were prepared by solid-state synthesis from high purity (>99.99\%) starting materials: $\mathrm{La}_{2} \mathrm{O}_{3}$ (prefired in flowing $\mathrm{O}_{2}$ at $1000^{\circ} \mathrm{C}$ for $12 \mathrm{hrs}$ ), $\mathrm{MnO}_{2}$, and $\mathrm{SrCO}_{3}$. After several firings at 1000 $1350^{\circ} \mathrm{C}$, the powders were isostatically pressed into rods suitable for zone melting. The growth atmosphere was $20 \% \mathrm{O}_{2}$. In each case, the crystals grew with the c-axis normal to the zone travel direction. The resulting highly textured polycrystalline boules can be cleaved readily to yield shiny black crystals of layered manganite. Typical dimensions of the cleaved crystals are $2 \times 2 \times 0.1 \mathrm{~mm}^{3}$. Back-reflection $\mathrm{x}$-ray Laue photographs establish that in all cases the cleaved crystals have the c-axis oriented normal to the thin plates. Detailed investigations of the atomic structure of these crystals using neutron diffraction show the good quality of our samples including the full occupancy of all lattice sites [13]. For the investigation of the microscopic structure, in particular the study of intergrowths, we performed TEM measurements on ion-milled platelets. Magnetization and susceptibility $(\chi)$ measurements were made on both, a SQUID and an extraction magnetometer from Quantum Design, equipped with 7 and $9 \mathrm{~T}$ superconducting solenoids, respectively. The trapped flux in the solenoids $(\sim 10 \mathrm{Oe})$ was monitored and the field values given are correct to within \pm 0.2 Oe. Measurements were made with the applied field either parallel to the ab-plane $(\mathrm{H} \| \mathrm{ab})$ or parallel to the c-axis (H II c). Resistivity measurements were reported previously [23].

\section{Intergrowth Structures}

Seshadri et al. have demonstrated the presence of intergrowths in these layered materials, using transmission electron microscopy (TEM) [15]. Intergrowths can be due to missing or extra layers of $\mathrm{SrO}$ atoms between the $\mathrm{MnO}_{6}$ octahedral planes. They can be envisioned as being generated to locally preserve the stoichiometry in the presence of concentration inhomogeneities. Such defects represent $n \neq 2$ variants of the RuddlesdenPopper series. Figure 6 displays high-resolution TEM images from samples with $\mathrm{x}=0.4$. The most prominent features in these pictures are the white bands, which form a regular 
array of horizontal lines. A detailed analysis reveals that these white bands are associated with the insulating SrO double layers, which can be seen from the inset. So, the imperfections seen in Fig. 6 correspond to missing or extra SrO layers. In Fig. 6a, the regular series of white bands is interrupted by an $n=5$ intergrowths structure, whereas in Fig. $6 \mathrm{~b}$, an extra white band is present which divides the regular units cell into two $n=1$ intergrowths features. Such intergrowths are also observed in the $\mathrm{x}=0.3$ samples as can be seen from Fig. 7 where two bright-field micrographs are shown. Here, the detailed atomic structure of the intergrowths is not visible due to the lower resolution. However, the intergrowths can still be identified as sharp vertical lines, perpendicular to the $c$-axis of the crystal (Fig. 7b). In Fig. 7a, no intergrowths are detectable, which means that the depicted segment of this crystal exhibits perfect $n=2$ bilayer periodicity. We find in general that the density of intergrowths can vary throughout the sample.

The intergrowth structures influence the magnetic properties. of $\mathrm{SrO}\left(\mathrm{La}_{1}\right.$ $\left.{ }_{x} \mathrm{Sr}_{x} \mathrm{MnO}_{3}\right)_{2}$ because they represent inclusions of a different variant of the RuddlesonPopper series and therefore a different magnetic entity. As already mentioned, the magnetic properties of the layered materials depend strongly on $n$ and therefore it is reasonable to anticipate that the intergrowths will also differ from the adjacent "perfect". layers. Thus, it is important to characterize the magnetic properties of the intergrowths as well as its influence on the general behavior of the layered material. An estimate of the intergrowth volume fraction can be made from magnetization measurements. We obtain a volume fraction of $0.1-1 \%$ intergrowths in the investigated composition range $\mathrm{x}=0.3$ 0.5 .

\section{Magnetic Properties}

Before we discuss the influence of the intergrowths on the magnetic properties, it is necessary to analyze the basic properties of the $\mathrm{SrO}\left(\mathrm{La}_{1-x} \mathrm{Sr}_{x} \mathrm{MnO}_{3}\right)_{2}$ host material itself. Figure 8 shows the measured magnetic moment for a $x=0.4$ sample in a relatively small applied field as a function of temperature. Here, we see the behavior of a typical ferromagnet, showing a substantial magnetic moment in a moderate external magnetic field up to $T_{C}$ where the ferromagnetic order breaks down. For $x>0.32$, the ab-plane is the easy plane of the magnetization with a weak four-fold anisotropy, whereas samples 
with $\mathrm{x}=0.30-0.32$ yield an out-of-plane orientation of the magnetization. The magnetocrystalline anisotropy is obvious from Fig. 9 where low temperature $\mathrm{M}(\mathrm{H})$ measurements of such samples are shown for different orientations of the externally applied field. In fig. 9, one also observes a step-like onset behavior for the c-axis $\mathrm{M}(\mathrm{H})$ curve of the $x=0.30$ sample. This $M(H)$ anomaly occurs at $H \approx 1 \mathrm{kOe}$ and is caused by the weak antiferromagnetic inter-layer coupling which has to be overcome first, after which we observe the conventional linear magnetization increase with applied field. At the onset field the sample actually undergoes a phase transition, the spin-flop transition which has recently been investigated in detail [26].

Besides understanding the basic magnetic properties in terms of the dominant magnetic interactions, $\mathrm{SrO}\left(\mathrm{La}_{1-\mathrm{x}} \mathrm{Sr}_{x} \mathrm{MnO}_{3}\right)_{2}$ provides another challenge associated with materials issues. As appears in Fig. 8, the low-field magnetization shows a transition at $\mathrm{T}_{\mathrm{C}}$, but the magnetization does not vanish or even approach $\mathrm{M}=0$ for $\mathrm{T}>\mathrm{T}_{\mathrm{C}}$ (The data shown here were measured on samples with particularly strong intergrowths content to emphasize their specific magnetic signature.). This can be seen in more detail in Fig. 10, where only the temperature range near $T_{C}$ is depicted. In Fig. 10a, we see the measured magnetic moment as a function of $\mathrm{T}$ for several values of $\mathrm{H}$. The observed data for large fields are consistent with the expected behavior of a field-induced magnetic moment even above $T_{c}$. This field induced moment should decrease with increasing temperature as one can see in the case of $\mathrm{H}=5 \mathrm{kOe}$. For low fields, however, the observed behavior is surprising because even for fields as low as $0.2 \mathrm{kOe}$ a significant magnetic moment is observed even $20 \mathrm{~K}$ above $\mathrm{T}_{\mathrm{C}}$. Furthermore, the moment seems to be almost independent of temperature as one moves further away from $\mathrm{T}_{\mathrm{c}}$. This unusual behavior becomes even more evident in Fig. 11b where we plot the inverse susceptibility $v s$ temperature. Here, one would expect the curves taken for various field strengths to disagree only in the vicinity of $\mathrm{T}_{\mathrm{C}}$ but approach each other for higher temperatures. This is obviously not the case and therefore, the observed magnetization measurements cannot be interpreted in terms of a field-induced magnetization above $\mathrm{T}_{\mathrm{c}}$. Instead, these results demonstrate the existence of a second magnetic entity with a higher ordering temperature. As already outlined in our discussion of the intergrowth structure, we would expect the intergrowths to have different magnetic properties and it seems reasonable to assume that an $n>2$ 
intergrowth structure should have an increased $\mathrm{T}_{\mathrm{c}}$. Thus, we associate this second, minor magnetic entity in our samples with intergrowths. An additional argument for this explanation is the fact that the residual magnetization above $T_{C}$, as a fraction of the total magnetic moment of the entire sample, varies from specimen to specimen, clearly indicating an extrinsic origin for the residual magnetization. We should also keep in mind that we saw such a strong variation for the density of intergrowths structures in Fig. 7.

To establish the connection between the residual magnetization and the intergrowths, we have also studied the field and temperature dependent ac-susceptibility. Due to their enhanced sensitivity, ac-susceptibility measurements allow for a much better separation of the intergrowth magnetization from the surrounding host signal, and enable us to track its properties even for temperatures below $T_{C}$ where the bulk ordering has already set in and dominates magnetization measurements. However, one has to realize, that the tracking of the intergrowth magnetization below $T_{C}$ only works because the easy axis of magnetization for bulk and intergrowths are perpendicular to each other. Figure 11a shows field dependent susceptibility curves $\chi(\mathrm{H})$ for three different temperatures measured on an $x=0.30$ sample. Due to the fact that all three measurements are relatively close to the bulk ordering temperature $T_{N}=74 \mathrm{~K}$, we find rather strong changes of the measured susceptibility curves with temperature. However, there is a common feature in all these curves, i.e. a sharp peak centered at $\mathrm{H}=0$. Thus, this signal corresponds to a very soft magnetic entity, which is obviously not correlated with the ordering temperature of the surrounding material. Detailed analysis reveals that the area under these peaks is in quantitative agreement with the residual magnetization above $T_{C}$ for each individual sample. Therefore, one can consistently interpret these anomalous sharp susceptibility peaks as being originated by the intergrowth magnetization. More detailed low field measurements are shown in Fig. $11 \mathrm{~b}$ for an extended $\mathrm{T}$ range. The data exhibit only rather weak $T$ dependence. Especially, one can clearly see that there are no substantial changes for temperatures near the bulk ordering temperature. Furthermore, we find a small but noticeable hysteresis in our data even for $\mathrm{T}=120 \mathrm{~K}$, which is far above the bulk ordering temperature. Thus, the signal can only originate from a ferromagnetically ordered entity, consistent with our interpretation as intergrowth magnetism. Our measurements are not consistent with the interpretation of the enhanced 
low-field susceptibility as a build-up of ferromagnetic fluctuations, which was suggested in the literature [10].

Figure 12 shows the observed magnetic moment as a function of $\mathrm{T}$ for an $\mathrm{x}=0.3$ sample. As we can see, even at room temperature, $i . e$. at $\mathrm{T}$ values three times as large as the $T_{C}$ of the host material, we still find a significant magnetic moment with only a very small magnetic field àpplied. In addition, we observe that the $\mathrm{M}(\mathrm{T})$ behavior shows three individual transition-like features. This highly unusual temperature dependence indicates that there are at least three different types of additional minor phases in this sample. This is in agreement with our interpretation because one would expect a variety of intergrowth structures with different $n$-values or spacial extents to be present. Figure 13 shows the field dependence of the magnetization for the $x=0.3$ sample at $250 \mathrm{~K}$ for in-plane as well as out-of-plane orientation of $\mathrm{H}$. Interestingly, one finds the residual magnetization to be oriented within the ab-plane as opposed to an orientation along the c-axis found below $\mathrm{T}_{\mathrm{C}}$ for this very sample. Thus, the preferred magnetization orientation changes at $T_{C}$, which can be explained within our picture of intergrowth magnetism being an independent magnetic entity. Our earlier interpretation of the preferred ab-orientation of the intergrowth magnetization in terms of the demagnetizing factor is not applicable [23]. Even though the demagnetizing factor will prefer the ab-plane orientation of the magnetization for $\mathrm{T}>\mathrm{T}_{\mathrm{C}}$, this argument does not apply for $\mathrm{T}<\mathrm{T}_{\mathrm{C}}$ as the host material is then polarized along the c-axis. The measurements in Fig. 11 show that the intergrowth magnetization stays in the ab-plane. Thus, the magnetocrystalline anisotropy must be responsible for the orientation and not the demagnetizing factor.

\section{Summary and Outlook}

The two-layered Ruddlesden-Popper phase $\mathrm{SrO}\left(\mathrm{La}_{1-\mathrm{x}} \mathrm{Sr}_{\mathrm{x}} \mathrm{MnO}_{3}\right)_{2}$, with $\mathrm{x}=0.3-$ 0.4 , exhibits $C M R$ near $T_{C}$ and a magnetic anisotropy which is strongly compositiondependent. The magnetic order observed for $\mathrm{T}>\mathrm{T}_{\mathrm{C}}$ includes not only the field induced or short-range order response of the intrinsic material, but a ferromagnetic signal due to structural imperfections known as intergrowths. These intergrowths are two-dimensional lattice defects and are visible in TEM images with an orientation parallel to the ab-planes. The intergrowths exhibit a magnetization orientation within the ab-plane even in the $\mathrm{x}=$ 
0.3 and $\mathrm{x}=0.32$ material, despite the fact that the $\mathrm{c}$-axis is the magnetocrystalline easy axis for the intrinsic host material at these compositions.

The intergrowth structures and the associated magnetism are significant in two aspects. First, they are essentially isolated magnetic entities for temperatures above $T_{C}$ and allow investigations of $2 \mathrm{D}$ ferromagnetism in a system with a non-conventional exchange mechanism. They are also an example of a 2D ferromagnetic material buried inside a host with an exchange enhanced magnetic susceptibility, which makes them an interesting research topic. Secondly, the intergrowth magnetism might play an important role for the magnetic properties of the host material in the vicinity of $T_{C}$. Here, the layered $\mathrm{SrO}\left(\mathrm{La}_{1-\mathrm{x}} \mathrm{Sr}_{\mathrm{x}} \mathrm{MnO}_{3}\right)_{2}$ is not a simple bulk phase near its ordering temperature, but rather a binary system of a majority phase weakly exchange-coupled to a ferromagnetic minority phase.

\section{Acknowledgments}

This work has been supported by the U. S. Department of Energy, Basic Energy Sciences - Materials Sciences under Contract W-31-109-ENG-38. 


\section{References}

[1] for a recent review, see for example: Y. Tokura and Y. Tomioka, JMMM 200, xxx (1999)

[2] C. Zener, Phys. Rev. 82403 (1951).

[3] P. W. Anderson and H. Hasegawa, Phys. Rev. 100, 675(1955)

[4] P. G. de Gennes, Phys. Rev. 118, 141 (1960)

[5] M. Imada, A. Fujimori, and Y. Tokura, Rev. Mod.Phys. 70, 1039 (1998)

[6] A. Millis, P. B. Littlewood, and B. I. Shraiman, Phys. Rev. Lett. 74, 5144(1995)

[7] for a recent review, see Gupta, JMMM 200 (1999)

[8] Y. Moritomo, A. Asamitsu, H. Kuwahara, and Y. Tokura, Nature 380, 141 (1996)

[9] Y. Moritomo, Y. Tomioka, A. Asamitsu, Y. Tokura, and Y. Matsui, Phys. Rev. B51, 3297 (1995)

[10] T. Kimura, Y. Tomioka, H. Kuwahara, A. Asamitsu, M. Tamura, and Y. Tokura, Science 2741698 (1996).

[11] P. D. Battle, M. A. Green, N. S. Laskey, J. E. Millburn, P. G. Radaelli, M. J. Rosseinsky, S. P. Sullivan, and J. F. Vente, Phys. Rev. B 5415967 (1996).

[12]P. Laffez, G, Van Tendeloo, R. Seshadri, M. Hervieu, C. Martin, A. Maignan, and B. Raveau, J. Appl. Phys. 805850 (1996). 
[13]J. F. Mitchell, D. N. Argyriou, J. D. Jorgensen, D. G. Hinks, C. D. Potter, and S. D. Bader, Phys. Rev. B 5563 (1997).

[14] D. N. Argyriou, J. F. Mitchell, J. B. Goodenough, O. Chmaissem, S. Short, J. D. Jorgensen, Phys. Rev. Lett. 781568 (1997).

[15] R. Seshadri, M. Hervieu, C. Martin, A. Maignan, B. Domenges, B. Raveau, and A. N. Fitch, Chem. Mater. 91778 (1997)

[16] T. G. Perring, G. Aeppli, Y. Moritomo, and Y. Tokura, Phys. Rev. Lett. 78, 3197 (1997)

[17] C. D. Potter, Maribeth Swiatek, S. D. Bader, D. N. Argyriou, J. F. Mitchell, D. J. Miller, D. G. Hinks, and J. D. Jorgensen, Phys. Rev. B 5772 (1998)

[18] S. D. Bader, R. M. Osgood III, D. J. Miller, J. F. Mitchell, and J. S. Jiang, J. Appl. Phys. 836385 (1998).

[19] S. Rosenkranz, R. Osborn, J. F. Mitchell, L. Vasiliu-Doloc, J. W. Lynn, S. K. Sinha, and D. N. Argyriou, J. Apply. Phys. 837348 (1998).

[20] R. Osborn, S. Rosenkranz, D. N. Argyriou, L. Vasiliu-Doloc, J. W. Lynn, S. K. Sinha, J. F. Mitchell, K. E. Gray, and S. D. Bader, Phys. Rev. Lett. 81, 3964 (1998)

[21] M. Medarde, J. F. Mitchell, J. E. Millburn, S. Short, and J. D. Jorgensen, Phys. Rev. Lett. 83, 1223 (1999)

[22] L. Vasiliu-Doloc, S. Rosenkranz, R. Osborn, S. K. Sinha, J. W. Lynn, J. Mesot, O. Seeck, W. K. Lee, and J. F. Mitchell, accepted for publication (Phys. Rev. Lett.) 
[23] A. Berger, R. M. Osgood III, J. S. Jiang, D. J. Miller, J. F. Mitcheil,and S. D. Bader, Mat. Sci. Eng. B63, 133 (1999)

[24] J. F. Mitchell, unpublished

[25] A. Berger, J. F. Mitchell, and S. D. Bader, unpublished

[26] U. Welp, A. Berger, D. Miller, V. K. Vlasko-Vlasov, K. E. Gray, J. F. Mitchell, and S. D. Bader, unpublished 


\section{Figure Captions}

Fig. 1: Resistivity vs. temperature for $\mathrm{SrO}\left(\mathrm{La}_{1-\mathrm{x}} \mathrm{Sr}_{\mathrm{x}} \mathrm{MnO}_{3}\right)_{2}(\mathrm{x}=0.3)$ in zero field and at $\mathrm{H}$ $=9 \mathrm{~T}$ for (a) $\mathrm{j} \| \mathrm{ab}$, and (b) $\mathrm{j} \| \mathrm{c}$. The resulting magnetoresistance values are shown (thick line) on the right hand side. Measurements were made on separate samples, both of whom had $\mathrm{T}_{\mathrm{C}} \approx 90 \mathrm{~K}$; the minor difference between the $\mathrm{T}_{\mathrm{C}}$ 's was normalized out.

Fig. 2: Energy scheme for the $\mathrm{Mn}^{3+}$-ion $3 \mathrm{~d}$ orbitals for various atomic configurations: five-fold degeneracy for the free ion (left), splitting between the triple degenerate $t_{2 g}$ and double degenerate $e_{\mathrm{g}}$ orbitals for a symmetric $\mathrm{MnO}_{6}$-octahedron (middle), splitting of the $3 \mathrm{~d}$ orbital energies for a Jahn-Teller distorted $\mathrm{MnO}_{6}$-octahedron (right).

Fig. 3: Schematic of the double exchange mechanism between $\mathrm{Mn}^{3+}$ - and $\mathrm{Mn}^{4+}$-ions, mediated by the $\mathrm{O}^{2 \cdot}(2 \mathrm{p})$-electrons. The effective $e_{\mathrm{g}}$ electron hopping can only occur between neighboring $\mathrm{Mn}$-ions if the corresponding $\mathrm{t}_{\mathrm{gg}}$ electron spins have a parallel component.

Fig. 4: Diagram of the structure $\mathrm{SrO}\left(\mathrm{La}_{1-\mathrm{X}} \mathrm{Sr}_{\mathrm{x}} \mathrm{MnO}_{3}\right)_{\mathrm{n}}$ : the variable $n$ refers to the number of $\mathrm{MnO}_{6}$ octahedral layers in the structure. The atoms shown are the $\mathrm{La}, \mathrm{Sr}$ cations in between the $\mathrm{O}_{6}$ octahedra which surround the $\mathrm{Mn}$ atoms.

Fig. 5: schematic of the experimentally determined magnetic groundstates for $\mathrm{SrO}\left(\mathrm{La}_{1-}\right.$ $\left.{ }_{x} \mathrm{Sr}_{\mathrm{x}} \mathrm{MnO}_{3}\right)_{2}$ in the composition range $\mathrm{x}=0.30$ to $\mathrm{x}=0.50$.

Fig. 6: High-resolution TEM micrograph showing the detailed intergrowth structure (marked with a pointer) for the $\mathrm{x}=0.4$ composition: (a) intergrowth with $n=5$, (b) intergrowth structure corresponding to two $n=1$ unit cells.

Fig. 7: Bright-field TEM micrograph showing (a) perfect crystal (b) crystal with intergrowths. In (b) the intergrowths are visible as vertical streaks in the photograph (marked with pointers). 
Fig. 8: Magnetic moment $v s$. temperature for a $\mathrm{SrO}\left(\mathrm{La}_{1-x} \mathrm{Sr}_{\mathrm{x}} \mathrm{MnO}_{3}\right)_{2}$ sample with $\mathrm{x}=0.4$ $(\mathrm{H}=0.5 \mathrm{kOe} / \mathrm{ab})$.

Fig. 9: Magnetization (normalized to the saturation magnetization $M_{s}$ ) vs. applied field $H$ for (a) $\mathrm{x}=0.4$ (b) $\mathrm{x}=0.3$ ( $\mathrm{H} \|$ ab: filled symbols, $\mathrm{H} \| \mathrm{c}$ : clear symbols, the temperature was held constant at $\mathrm{T}=5 \mathrm{~K}$ in all measurements).

Fig. 10: Magnetic moment vs. temperature of a $\mathrm{SrO}\left(\mathrm{La}_{1-x} \mathrm{Sr}_{x} \mathrm{MnO}_{3}\right)_{2}(\mathrm{x}=0.4)$ sample measured for a certain number of field values $\mathrm{H}$ : (a) original data, (b) inverse DCsusceptibility $\chi^{-1}$ values deduced from the data.

Fig. 11: field-dependent AC-susceptibility data for various temperatures, measured on a $\mathrm{x}=0.30$ sample; (a) high-field data in the vicinity of $T_{\mathrm{N}}$ (bulk), (b) low field data for temperatures between $\mathrm{T}=20 \mathrm{~K}$ and $\mathrm{T}=200 \mathrm{~K}$.

Fig. 12: Magnetic moment $v s$. temperature for a $\mathrm{SrO}\left(\mathrm{La}_{1-x} \mathrm{Sr}_{\mathrm{x}} \mathrm{MnO}_{3}\right)_{2}$ sample with $\mathrm{x}=0.3$ $(\mathrm{H}=15 \mathrm{Oe} \| \mathrm{ab})$. Three different transitions at $\mathrm{T}_{\mathrm{i}}^{*}$ are identified.

Fig. 13: Magnetic moment $v s$. applied field for a $\mathrm{SrO}\left(\mathrm{La}_{1-\mathrm{x}} \mathrm{Sr}_{\mathrm{x}} \mathrm{MnO}_{3}\right)_{2}$ sample $(\mathrm{x}=0.3)$ at $\mathrm{T}=250 \mathrm{~K}$ with $\mathrm{H} \| \mathrm{ab}$ (filled symbols) and $\mathrm{H} \| \mathrm{c}$ (clear symbols). Both measurements are superimposed on a linear paramagnetic background from the intrinsic portion of the crystal. 


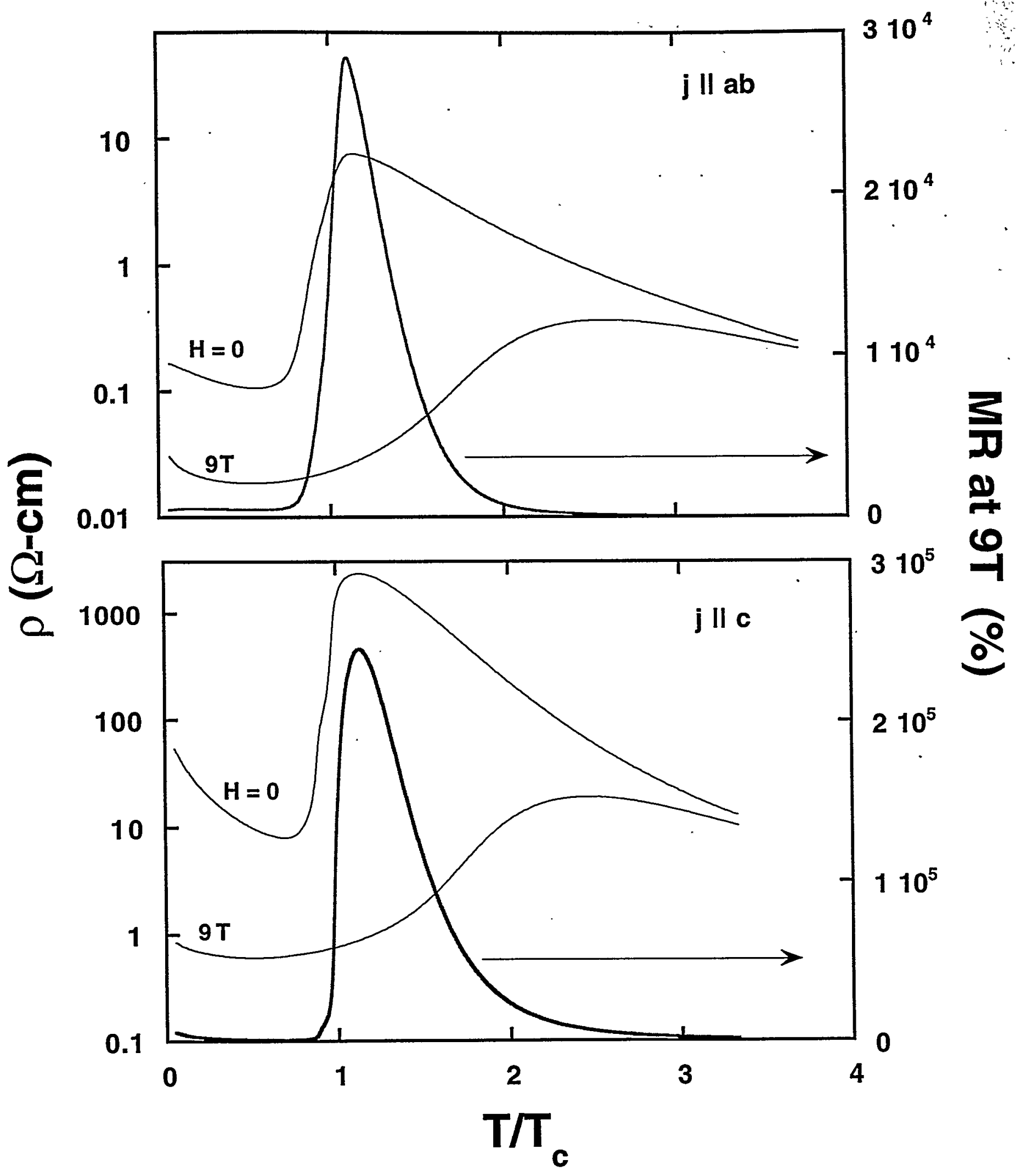

Fig. 1 


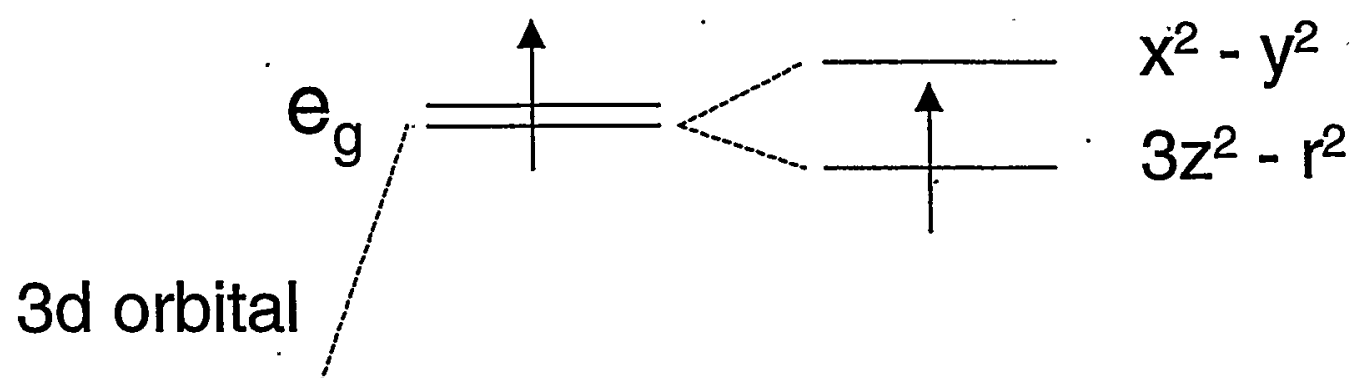

$\mathrm{Mn}^{3+}$
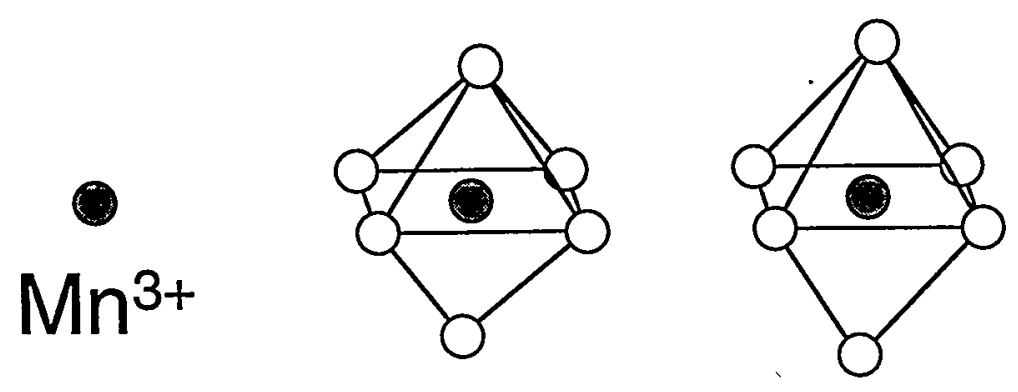

$x y$

$y z, z x$

Fig. 2 


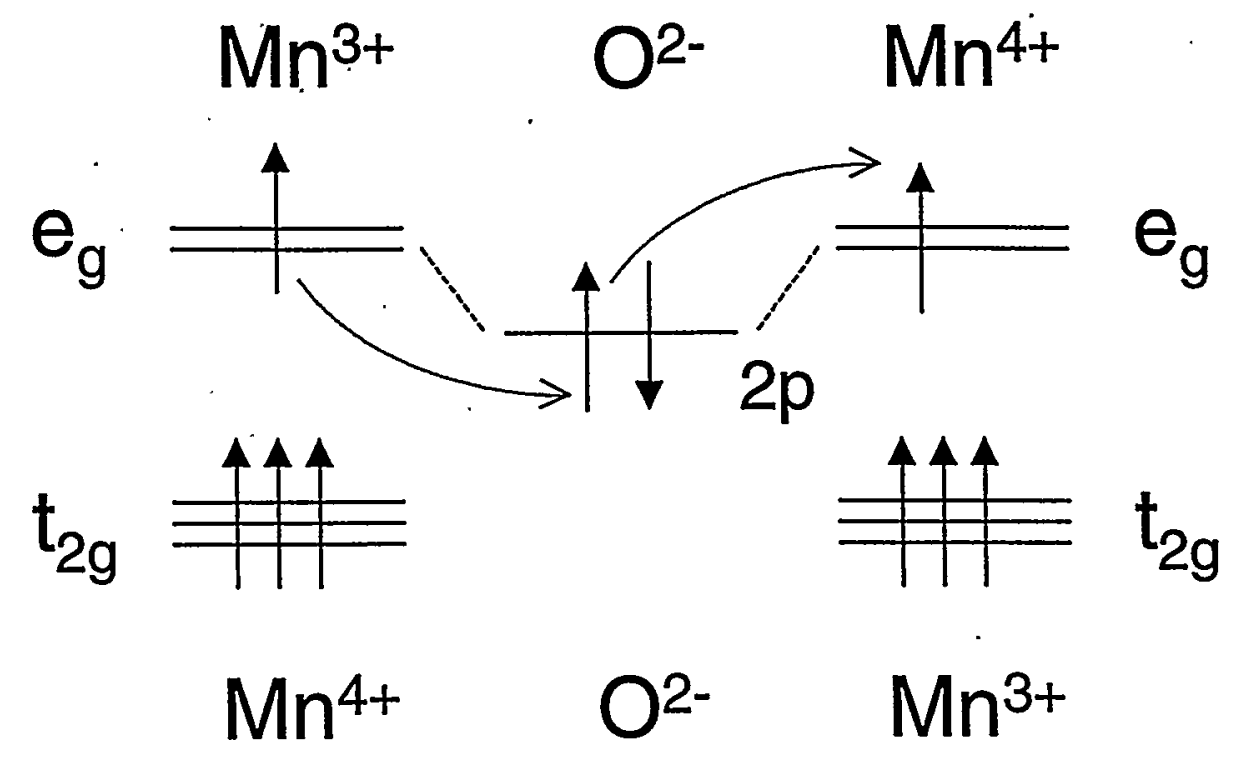

Fig. 3 


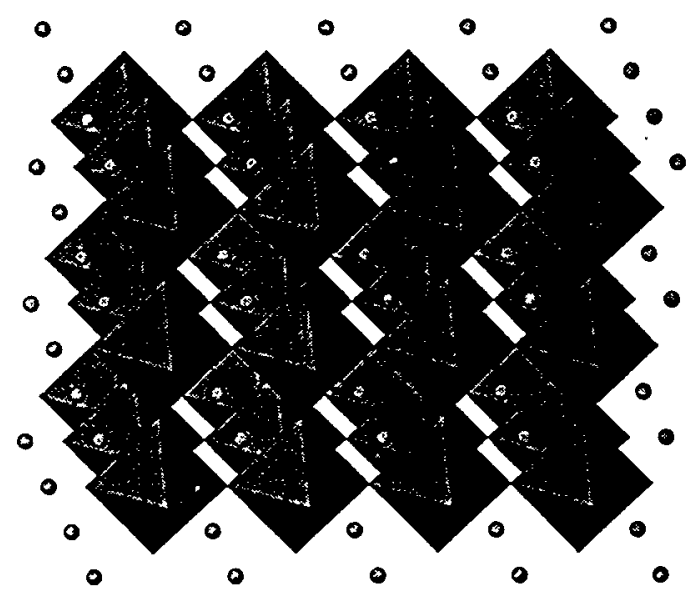

8

II

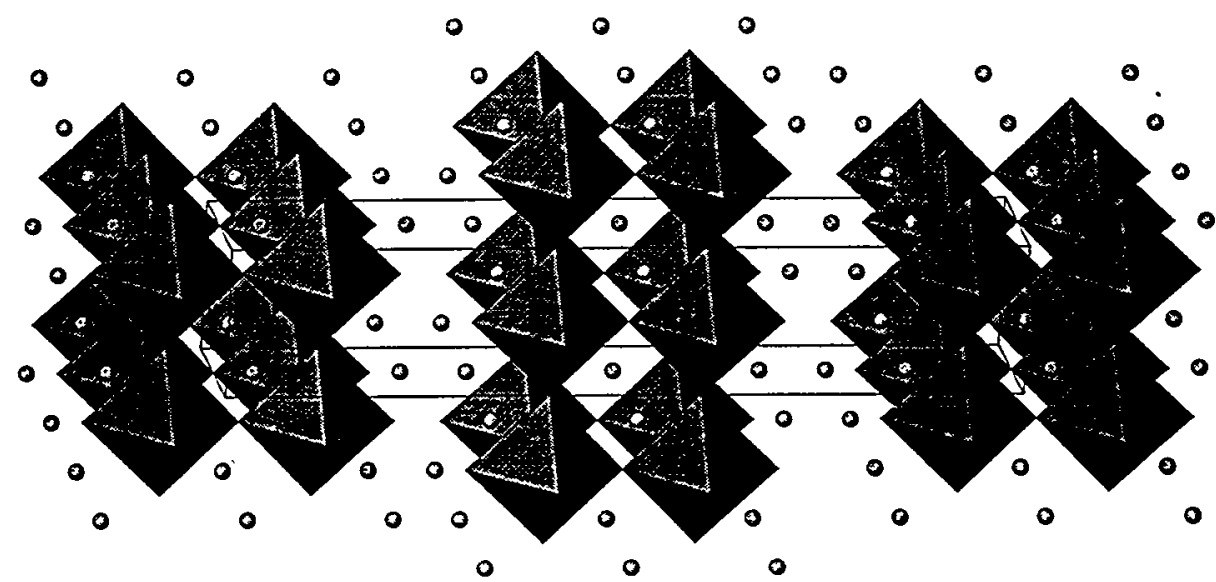

$N$

II
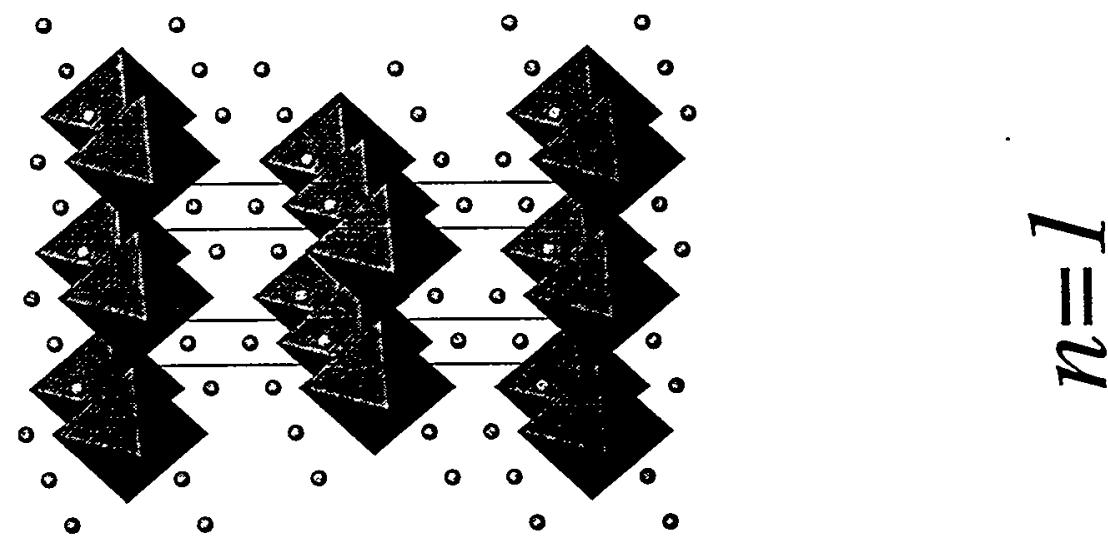


\section{$\operatorname{AFM}(\mathrm{c})$}
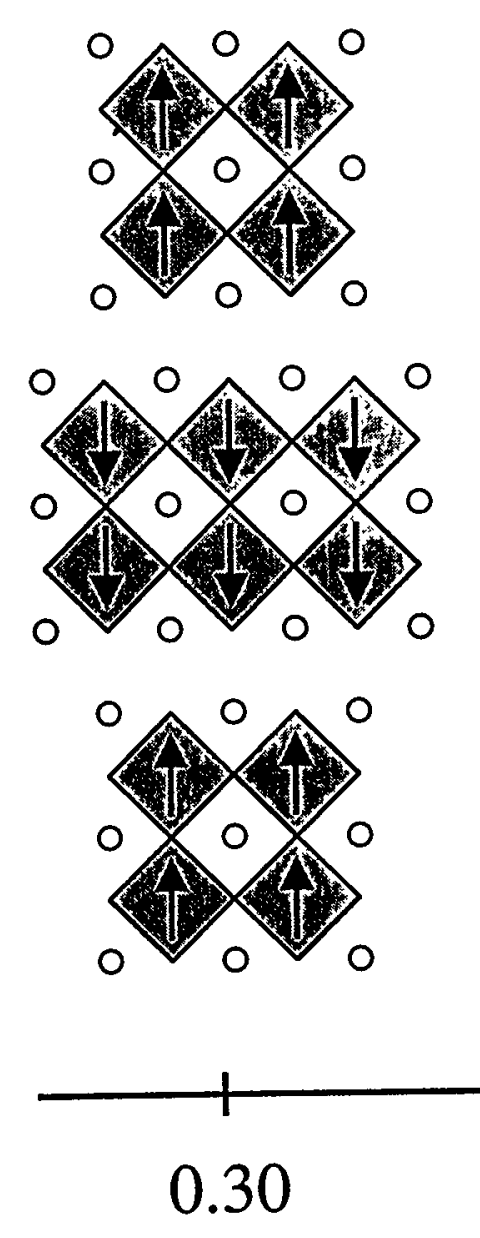
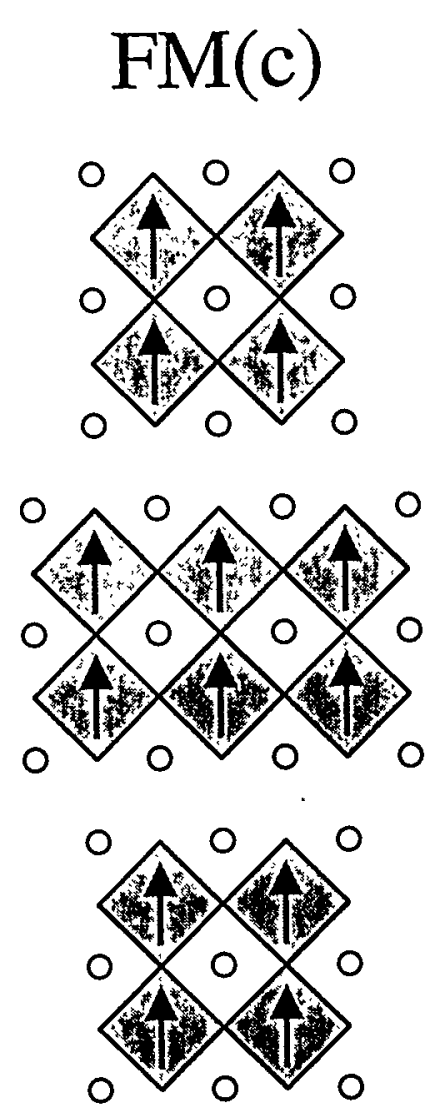

$\mathrm{FM}(\mathrm{ab})$
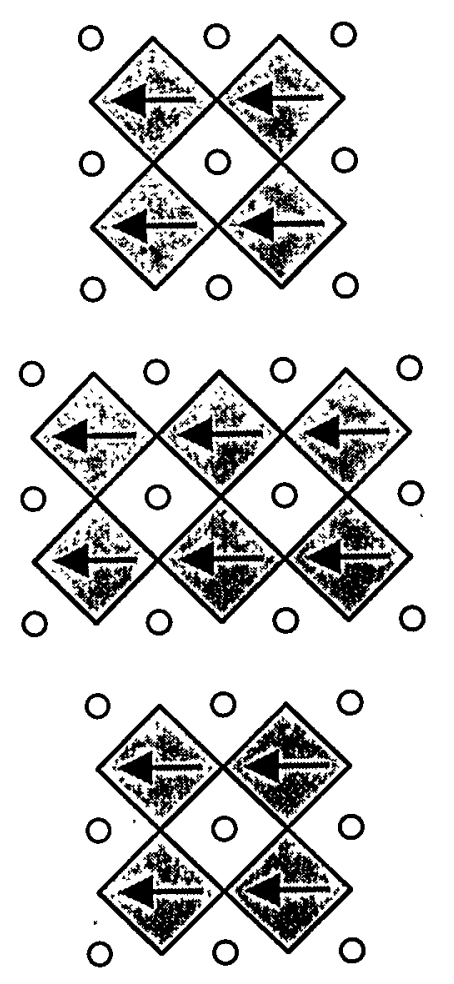

$\operatorname{AFM}(a b)$
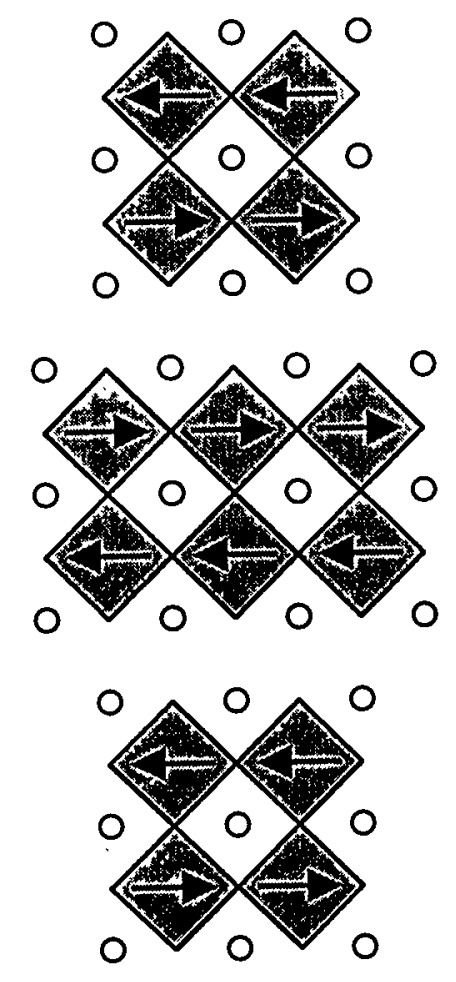

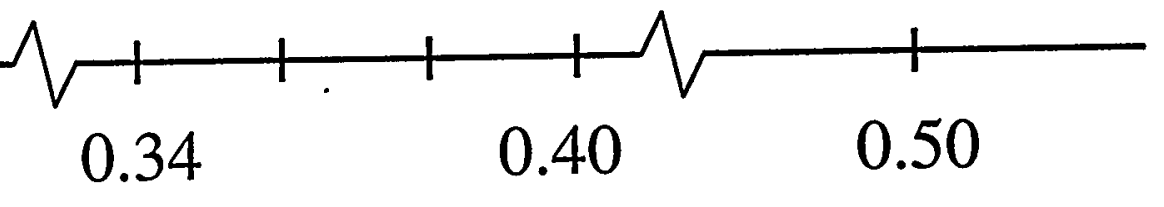

$\mathrm{Sr}$ - doping level $\mathrm{x}$ 


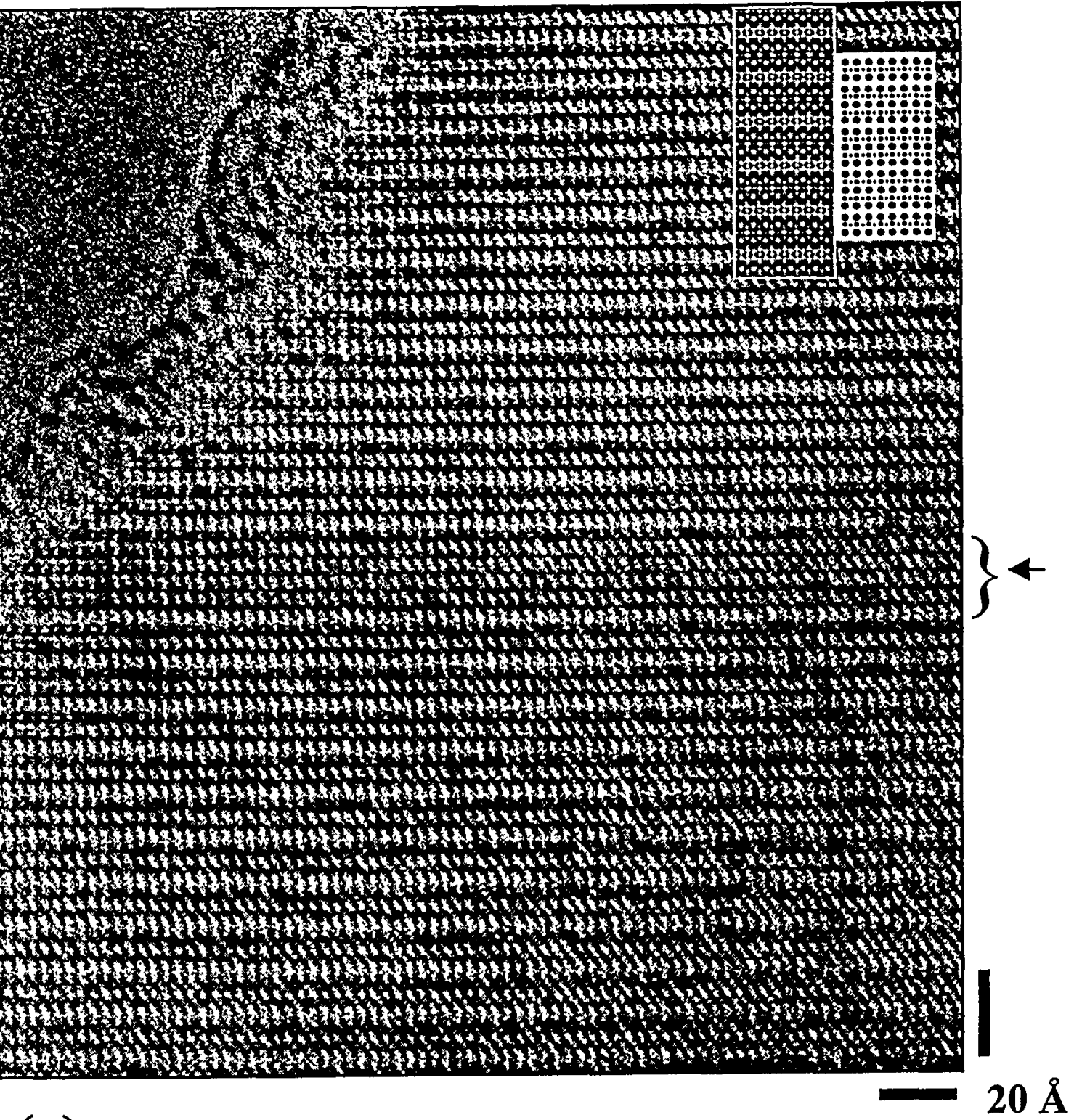

(a) 


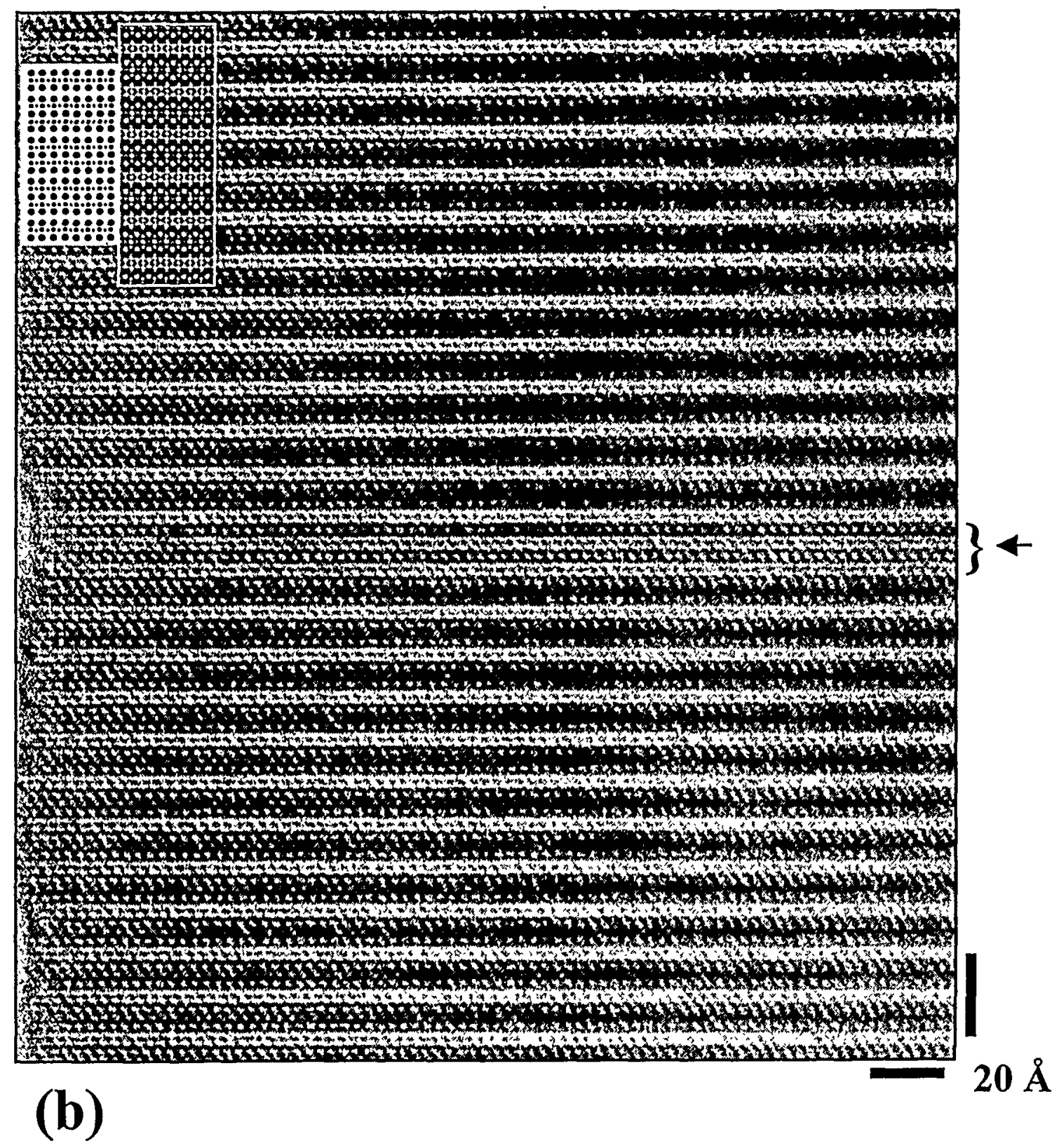



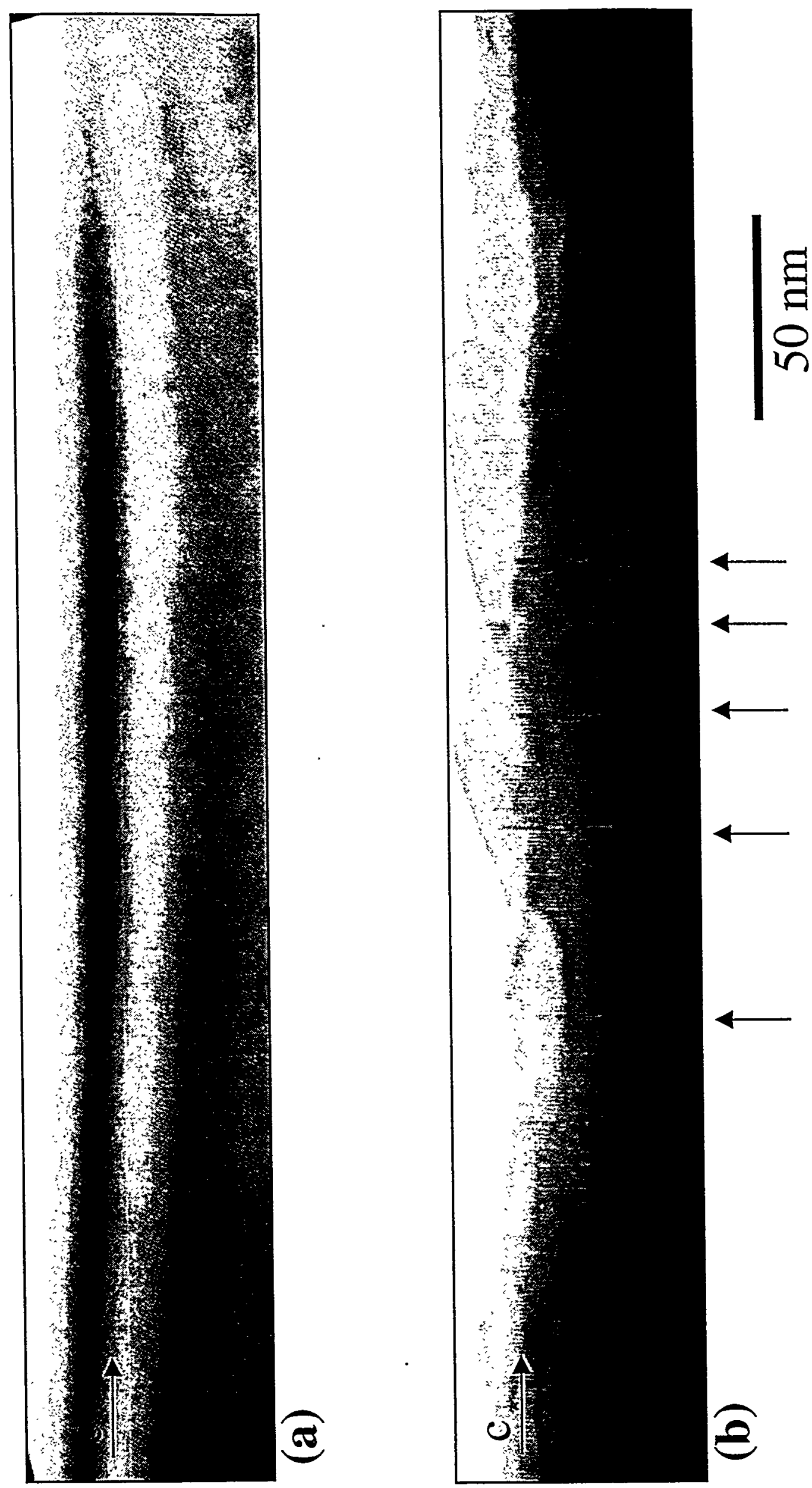

$\dot{4}$ 


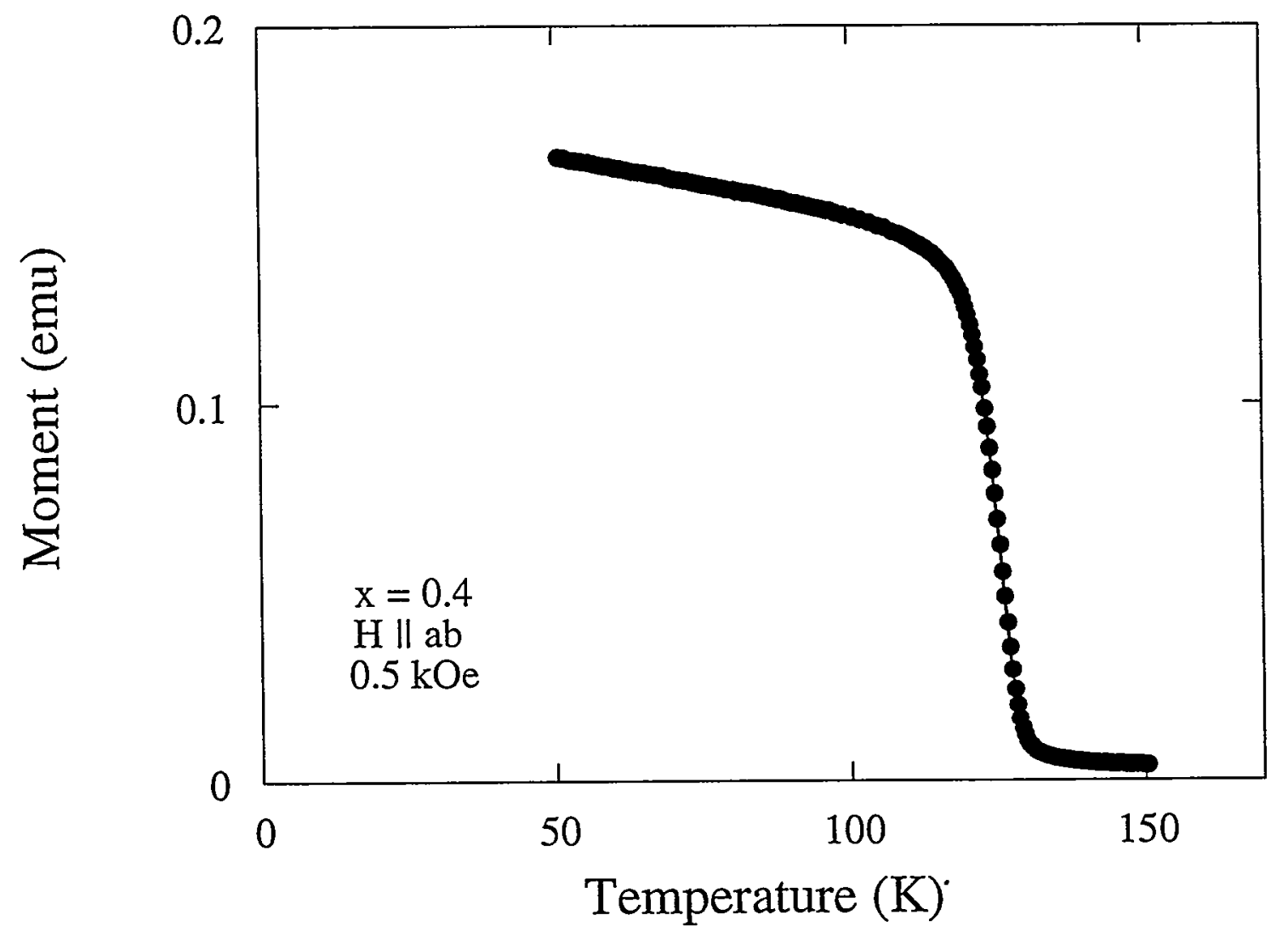

Fig. 8 

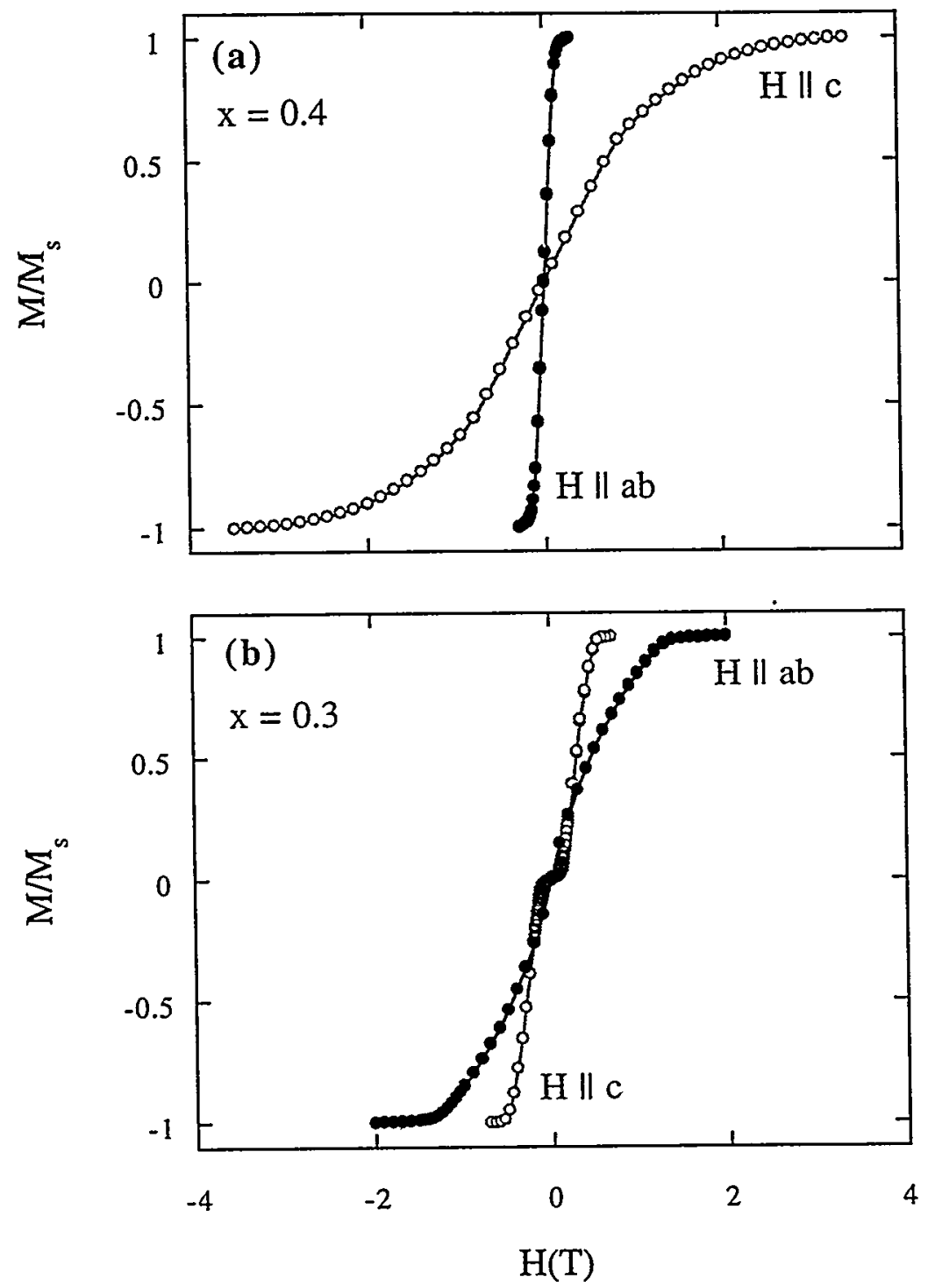

Fig. 9 

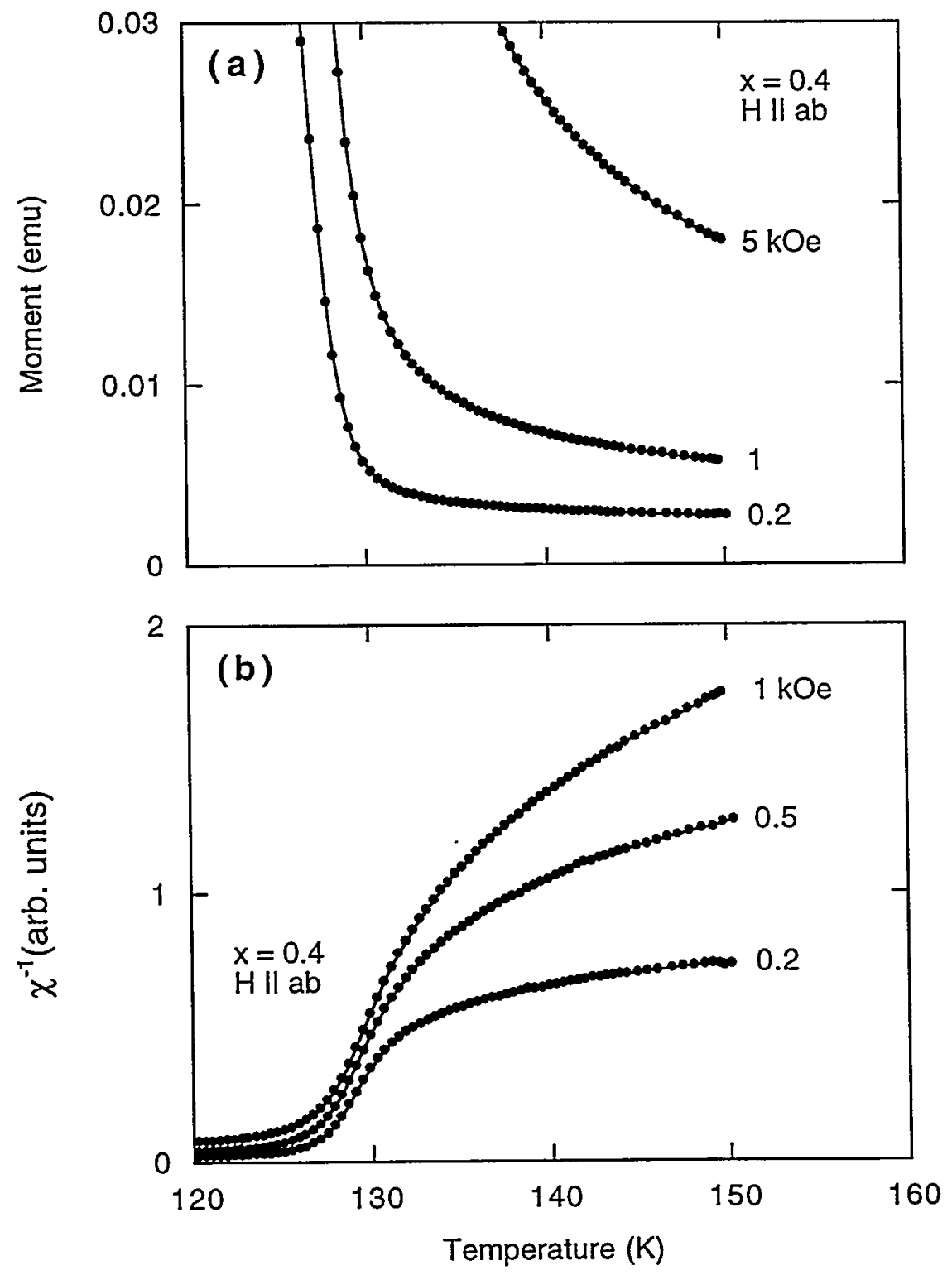

Fig. 16 


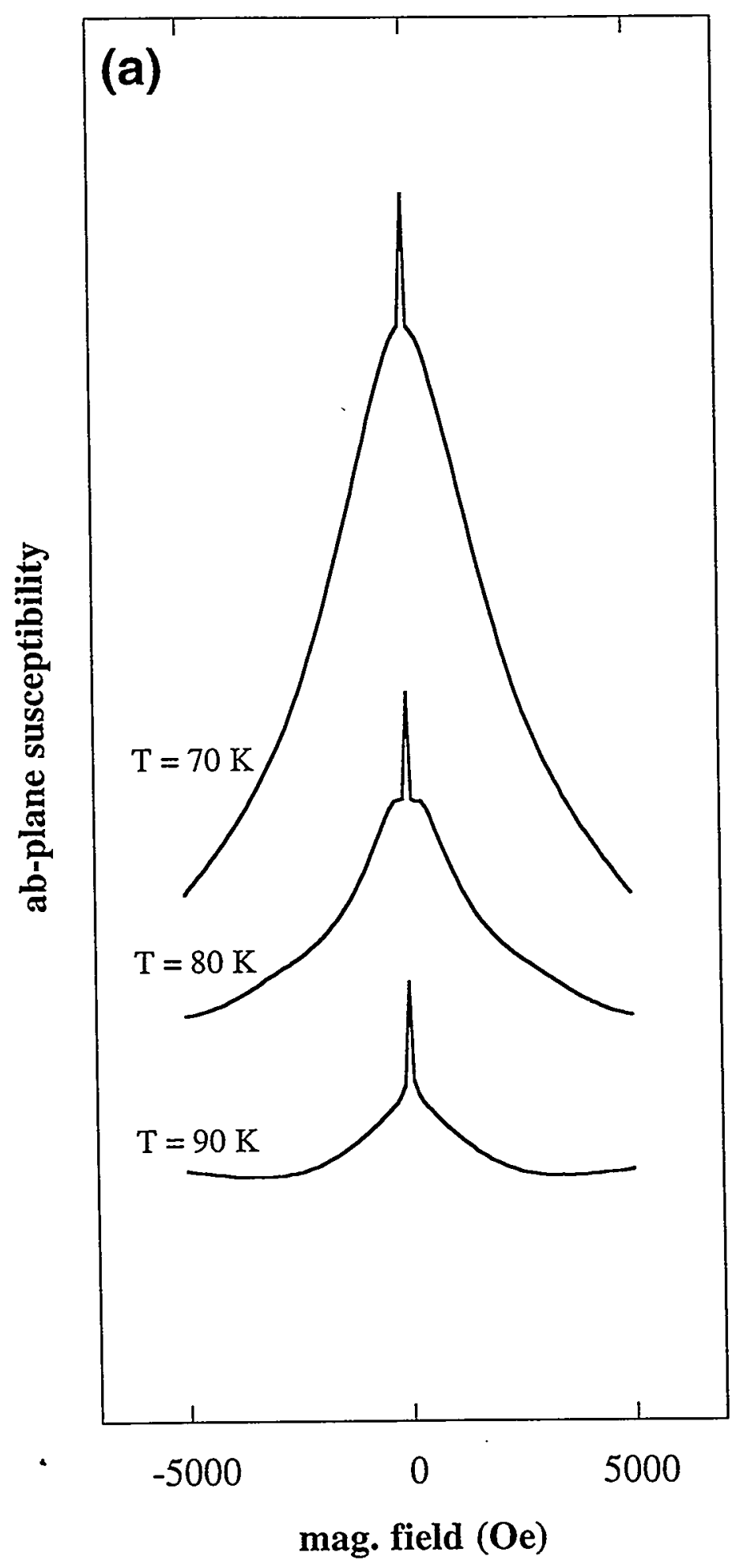

Fig. I/c 


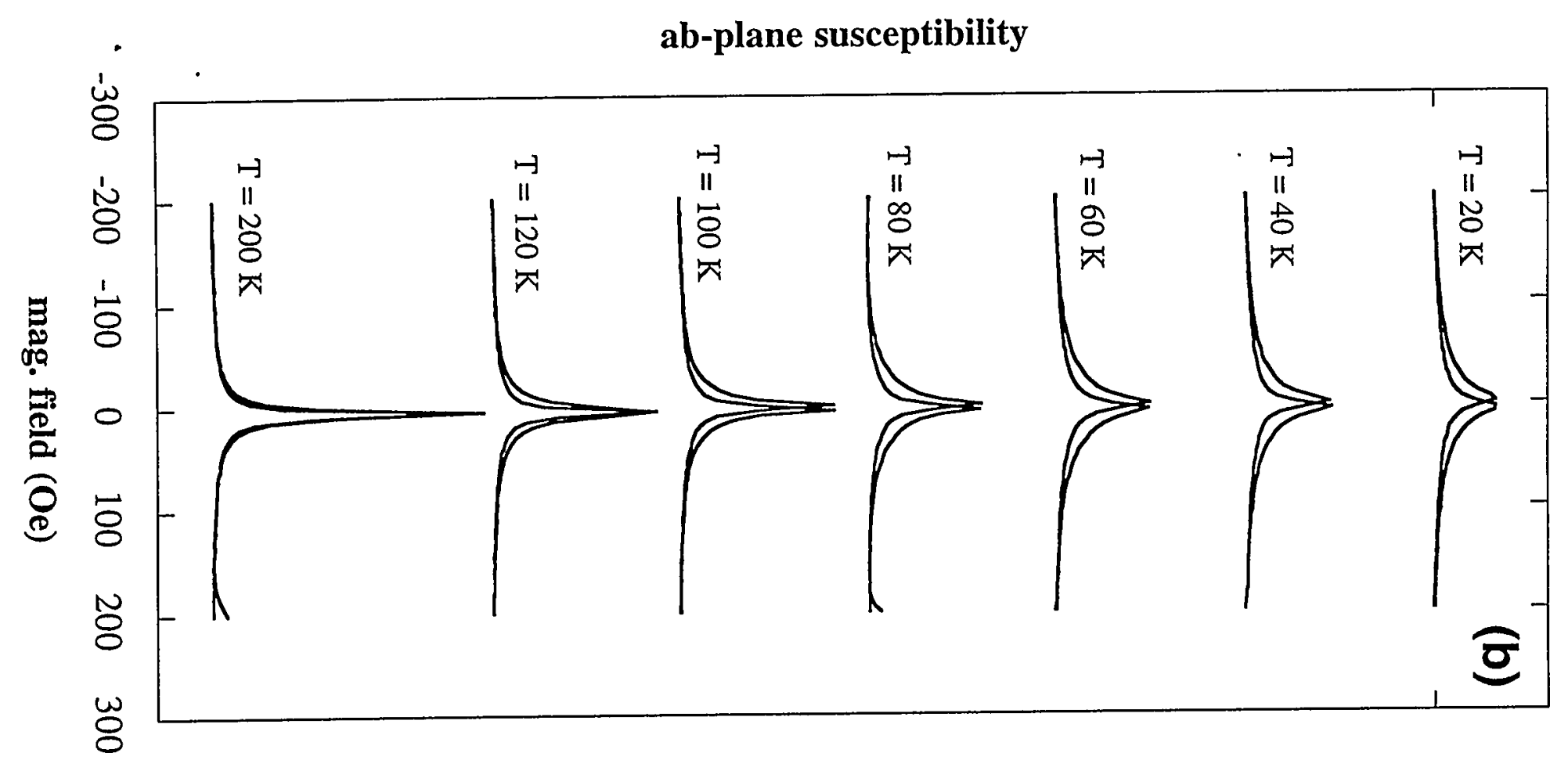

807
ते 


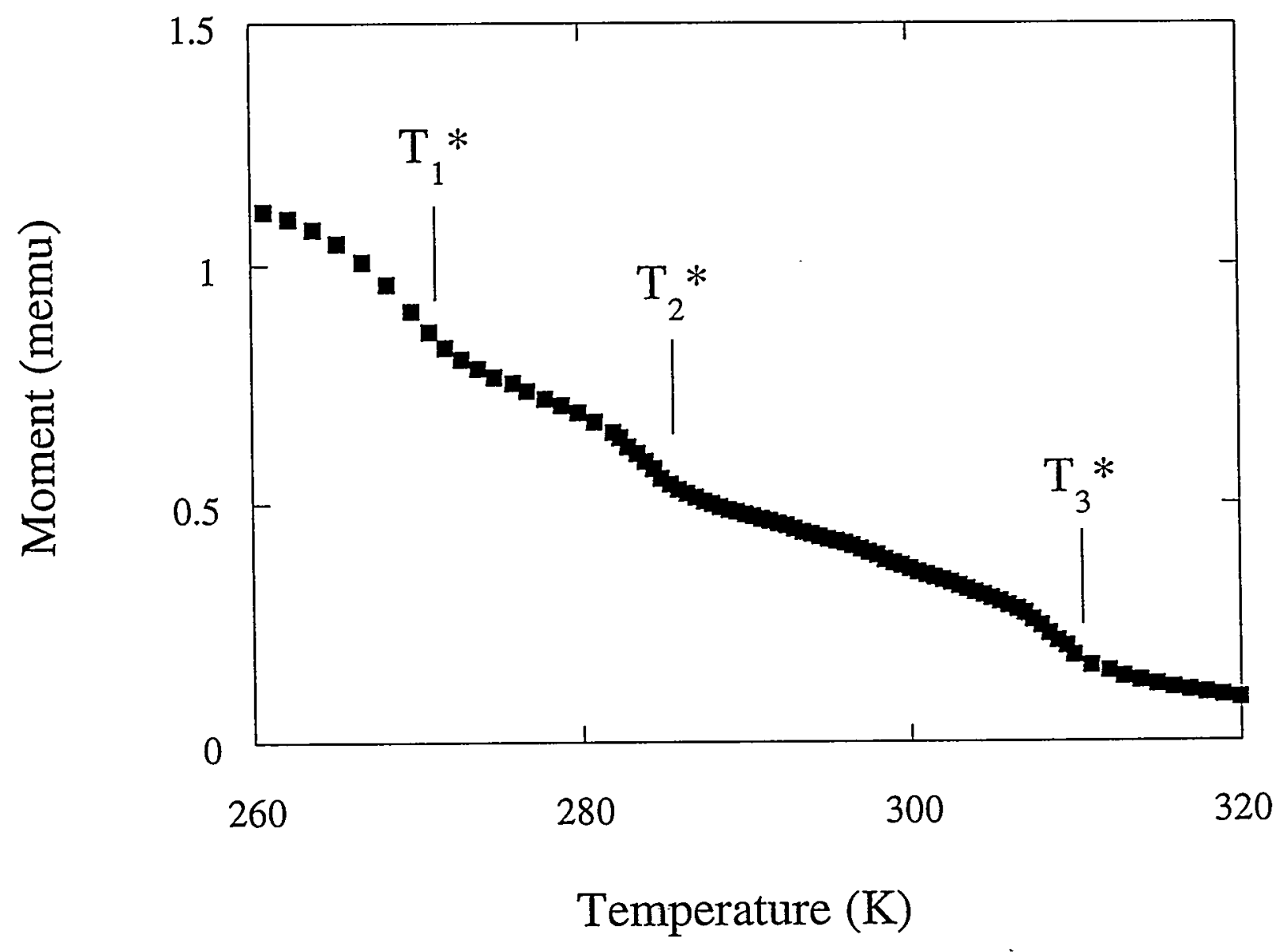

Fig.l. 


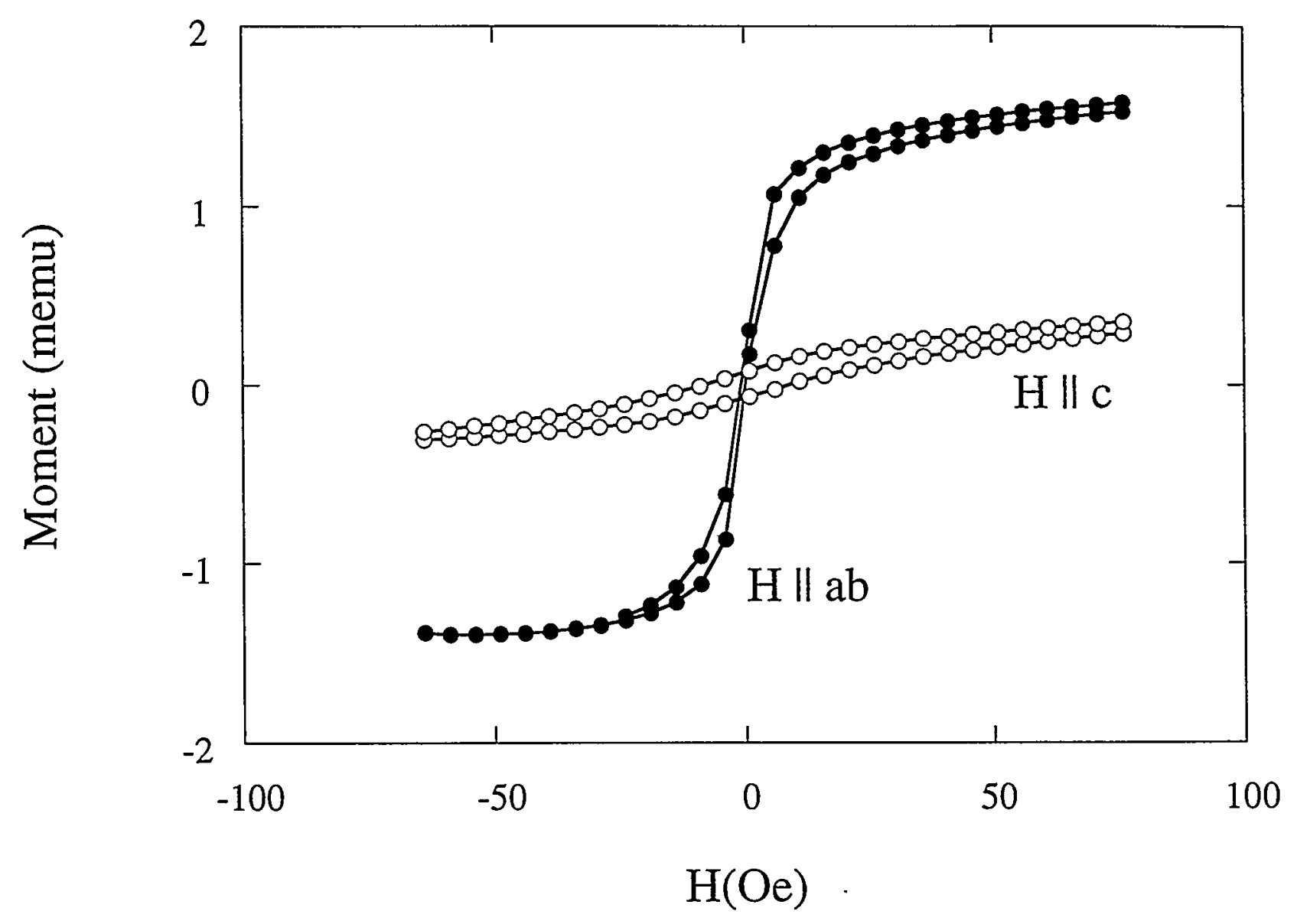

Fig. 1 